\title{
Classificação do Filme Lacrimal usando a Função K de Ripley como Descritor de Textura
}

\author{
Luana B. Cruz, José Denes L. Araújo, Jefferson A. Sousa, \\ João D. S. Almeida, Geraldo B. Júnior, Aristófanes C. Silva, Anselmo C. Paiva ${ }^{1}$ \\ ${ }^{1}$ Núcleo de Computação Aplicada - Universidade Federal do Maranhão (UFMA) \\ CEP 65080-805 - São Luís - MA - Brasil \\ \{luana.b.cruz, josedenes, jefferson.alves, jdallyson,
geraldo, ari, paiva\}anca.ufma.br
}

\begin{abstract}
Dry eye syndrome is a disease that affects great part of the population, making daily activities difficult. The diagnosis of this disease is a challenging task for ophthalmologists because of its multifactorial etiology. One of the most used tests consists of manually classifying tear film images, captured with the Doane interferometer. Therefore, an automated system to support specialists in the diagnosis becomes very useful. This work presents a method for automatic classification of the tear film lipid layer, using Ripley's $K$ function for feature extraction, and the classifiers Naive Bayes and Bayes Net. The proposed method achieved classification rates over $95 \%$.
\end{abstract}

Resumo. A síndrome do olho seco é uma doença que afeta grande parte da população dificultando suas atividades diárias. O diagnóstico desta doença é uma tarefa desafiadora para oftalmologistas, devido à sua etiologia multifatorial. Um dos testes mais utilizados consiste na classificação manual das imagens do filme lacrimal capturadas com o interferômetro Doane. Assim, torna-se útil o uso de um sistema automático para suporte ao diagnóstico pelos especialistas. Neste trabalho é proposto um método para a classificação automática da camada lipídica do filme lacrimal, usando a função $K$ de Ripley para extrair características e os classificadores Naive Bayes e Bayes Net. O método proposto apresenta taxas de classificação superiores a $95 \%$.

\section{Introdução}

O filme lacrimal é responsável por umidificar a superfície ocular sendo composto por lágrimas, secretadas pela glândula lacrimal, distribuídas através do movimento de piscar dos olhos [Remeseiro et al. 2014]. A sua estrutura é composta de várias camadas: a camada lipídica (externa), que previne a evaporação da água; camada aquosa (intermediária), que assegura a nutrição da córnea e protege de corpos estranhos; e camada mucosa (interna), responsável pela adesão das lágrimas aos olhos [Rolando and Zierhut 2001].

A síndrome do olho seco é uma doença multifatorial da superfície ocular que resulta em desconforto, distúrbios visuais e instabilidade do filme lacrimal. Apresenta como principais sintomas sensação de corpo estranho, queimação, olhos vermelhos, irritação nos olhos, fotofobia, embaçamento visual e lacrimejamento excessivo, que podem causar impacto na qualidade de vida. A prevalência da síndrome do olho seco na população 
mundial varia entre $6 \%$ e $34 \%$, sendo mais comum em adultos acima de 50 anos, e afeta principalmente as mulheres [Valim et al. 2015].

O diagnóstico da síndrome do olho seco é uma tarefa difícil devido à sua etiologia multifatorial e vários testes clínicos, que podem ser utilizados tanto para o diagnóstico quanto para o tratamento. A avaliação dos padrões de interferência que categorizam a camada lipídica, é um dos testes mais comuns para diagnosticar o olho seco. Essa avaliação é realizada utilizando o interferômetro Doane [Doane 1989], capturando imagens que permitem observar as mudanças ocorridas na camada lipídica. Em [Remeseiro et al. 2015] foi proposta uma classificação dessas imagens em cinco categorias: franjas fortes, coalescente de franjas fortes, franjas finas, coalescente de franjas finas e detritos.

Com os avanços da tecnologia da computação, por meio da natureza digital das imagens médicas geradas, a pesquisa e desenvolvimento de técnicas de processamento de imagem contribuíram para o surgimento de ferramentas CAD (Computer Aided Detection) e CADx (Computer Aided Diagnosis) de diversos tipos nos últimos anos. Os sistemas CAD e CADx ajudam a equipe médica na interpretação de imagens, aprimorando a eficiência e a precisão de diagnósticos e, consequentemente, proporcionam uma outra concepção [Doi 2007].

O objetivo deste trabalho é apresentar um novo método de classificação das categorias dos padrões de interferência da camada lipídica, em imagens interferométricas do filme lacrimal, aplicando uma abordagem de análise de textura. Como contribuição é apresentado o uso da função K de Ripley como descritor de textura, na seleção de características o algoritmo Greedy Stepwise e na classificação os algoritmos de redes bayesianas Naive Bayes e Bayes Net. As técnicas poderão ainda ser incorporadas a um sistema do tipo CADx e, portanto, contribuir para o aumento da produtividade e melhoria nas taxas dos diagnósticos.

Este artigo está organizado da seguinte forma: na Seção 2, são apresentados os trabalhos relacionados com o tema da pesquisa. Na Seção 3, é apresentada a função K de Ripley utilizada como descritor de textura. Na Seção 4, são descritos os materiais e métodos propostos. Na Seção 5, são apresentados os experimentos e resultados para validar nossa pesquisa, e um estudo comparativo com trabalhos equivalentes. Finalmente, as conclusões e trabalhos futuros são apresentados na Seção 6.

\section{Trabalhos Relacionados}

$\mathrm{Na}$ literatura estão disponíveis poucos trabalhos relacionados com o problema de diagnóstico das categorias da camada lipídica do filme lacrimal, utilizando imagens interferométricas capturadas pelo interferômetro Doane. A seguir, são apresentados resumos desses trabalhos.

No método proposto em [Villaverde et al. 2014] as imagens são submetidas a análise de textura utilizando filtro Butterworth, filtro de Gabor, transformada discreta de Wavelet, campos aleatórios de Markov [Çesmeli and Wang 2001] e características de matrizes de coocorrência. Essas características são extraídas das imagens em escala de cinza e no espaço de cor $\mathrm{L}^{*} \mathrm{a}^{*}$ b. Em seguida são utilizados os algoritmos de seleção de características baseada em correlação [Hall 1999], filtro baseado em consistência [Dash and Liu 2003] e INTERACT [Zhao and Liu 2007], com o objetivo de selecionar 
as características mais relevantes e reduzir o tempo de processamento. A classificação é realizada utilizando Máquina de Vetores de Suporte [Vapnik 1999]. O método alcançou resultados superiores a $91 \%$ de acurácia.

Em [Remeseiro et al. 2015] foi proposto um método que utiliza informações de cor e textura. As imagens são analisadas, em termos de cores, de três formas diferentes, utilizando-se a imagem em escala de cinza, no espaço de cor L*a*b e as cores oponentes do espaço de cor RGB. Em seguida, características de textura são extraídas, utilizando métodos baseados em processamento de sinais (filtro Butterworth, filtro de Gabor e a transformada discreta de wavelet), método baseado em modelo (campos aleatórios de Markov) e um método estatístico utilizando características de coocorrência. As características extraídas são classificadas utilizando os algoritmos de aprendizagem de máquina Naive Bayes, Random Tree, Random Forest e Máquina de Vetores de Suporte [Dean 2014]. O método apresentou taxas de acerto superiores a 93\%.

Os trabalhos relacionados anteriormente, indicam serem promissores em pesquisas a análise de textura para a extração de características de imagens interferométricas, com intuito de auxiliar no diagnóstico das categorias da doença olho seco. Neste trabalho, o objetivo é apresentar melhorias na descrição de padrões de texturas, com a aplicação da função K de Ripley e a utilização de classificadores para categorizar com precisão e consistência as imagens de interferometria da camada lipídica do filme lacrimal.

\section{Função K de Ripley}

Esta função é comumente utilizada em análise de dados espaciais, sendo frequentemente empregada em ecologia, para descrever a distribuição espacial de árvores e outras espécies em uma floresta. Nas últimas três décadas, sua aplicação também foi utilizada nas mais diversas áreas como: epidemiologia, geomorfologia, criminologia, geologia e etc [Lancaster and J Downes 2004].

Trata-se, portanto, de uma técnica para resumir um padrão de pontos, testar hipóteses sobre o padrão, estimar parâmetros e ajustar modelos. A função K de Ripley é definida pela Equação 1 [Ripley 1977]:

$$
R(d, i)=\sqrt{\frac{A k(i, j)}{N}}, i \neq j
$$

onde $d$ representa a função de distância usada na análise, $A$ representa a área da região em questão (calculado a partir do ponto de referência $i$ sob a distância $d$ ) e $k$ é a função de pertinência que verifica se $j$ está dentro do conjunto de análise determinado por $i$ e $d$.

Em suma, a função K de Ripley calcula uma relação do número de indivíduos de uma determinada espécie distribuída em uma região de estudo. Segundo [Martins 2007], além do uso convencional (círculos) da função K de Ripley, foi proposta também uma nova forma de aplicação da função, através da análise dos padrões de pontos em anéis. Basicamente, a modificação consiste em substituir a região de interesse (Figura 1 (a)) pela região compreendida entre dois círculos concêntricos (Figura 1 (b)). Além dessas abordagens, formas complexas também podem ser aplicadas.

O cálculo do raio de cada círculo, é realizado com base no raio máximo determinado pela região. Inicialmente, é necessário calcular a menor dimensão da Região 


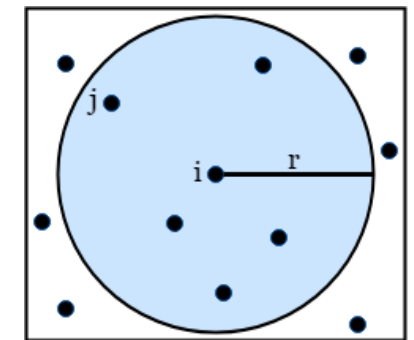

(a)

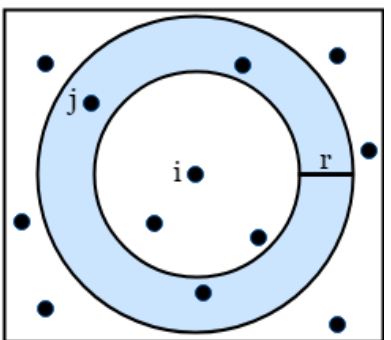

(b)

Figura 1. Abordagens da Função K de Ripley. (a) Círculos e (b) Anéis. Fonte: Autor.

de Interesse (ROI), assim é representado o raio máximo pela metade do valor da menor dimensão. A partir do raio máximo, calculam-se os demais através da alteração de proporção em relação ao primeiro (Equação 2).

$$
\text { Raio }_{i}=\frac{\text { Raio }_{\max }}{i}
$$

onde $i=1,2,3 \ldots, n$ e $n$ é a quantidade de raios determinados pela aplicação.

Para calcular a divisão em anéis, segue-se o mesmo princípio do cálculo da divisão em círculos. A diferença está no fato de anéis não possuírem áreas comuns entre si, como tem os círculos concêntricos. Assim, o raio máximo do anel interno é utilizado como um raio mínimo para o próximo.

A partir do mesmo cálculo do raio máximo obtido pela divisão de círculos, os raios mínimos e máximos dos anéis são obtidos usando a Equação 3:

$$
\begin{gathered}
\operatorname{Raio}_{\text {min }}(i)=\frac{\text { Raio }_{\text {max }}}{n} i \\
\operatorname{Raio}_{\text {max }}(i)=\frac{\text { Raio }_{\text {max }}}{n}(i+1)
\end{gathered}
$$

onde $i=1,2,3 \ldots, n$ e $n$ é a quantidade de raios determinados pela aplicação.

Portanto, as divisões criam novas representações de regiões circulares e concêntricas da região, a partir das ROIs das imagens. Assim, cada divisão gera recortes individualizados que irão passar por todo o processo de extração de características.

\section{Materiais e Métodos}

Nesta Seção, são descritos os procedimentos adotados para o desenvolvimento do método proposto para categorizar os padrões de interferência da camada lipídica, em imagens de interferometria do filme lacrimal. A Figura 2 apresenta as etapas seguidas pelo método proposto. Na primeira etapa, é feita a aquisição de imagens da base VOPTICAL_GCU [VOPTICAL_GCU 2017]. Então, sua ROI é extraída. Em seguida, é aplicada a extração de características usando a função K de Ripley, e o algoritmo Greedy Stepwise é utilizado para seleção de características. Finalmente, classificamos e validamos os resultados. 


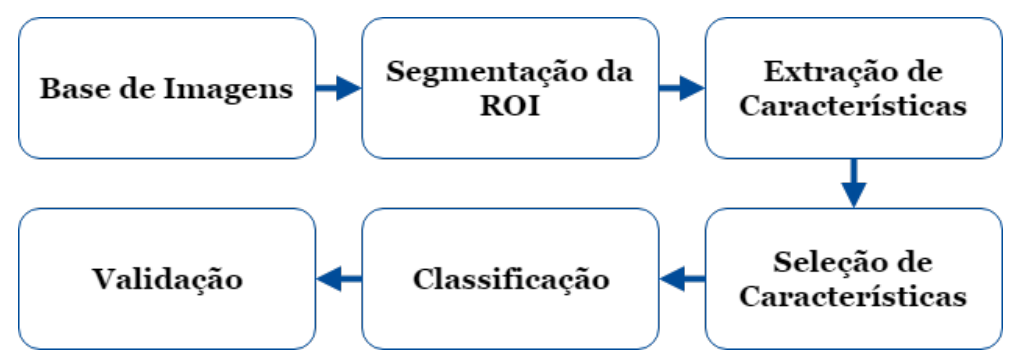

Figura 2. Etapas do método proposto.

\subsection{Base de Imagens}

A base de imagens utilizada é a VOPTICAL_GCU [VOPTICAL_GCU 2017], composta por imagens interferométricas do filme lacrimal, adquiridas de pacientes com olho seco com faixa etária entre 16 e 55 anos. Todas as imagens foram adquiridas por dois optometristas do Departamento de Ciências da Vida, Universidade Caledônia de Glasgow (Glasgow, UK).

A aquisição dessas imagens foi realizada com o interferômetro Doane [Doane 1989] e uma câmera digital CMEX-1301. As imagens possuem resolução 1280 x 1024 pixels no espaço de cores RGB. A base é composta por 106 imagens das cinco categorias consideradas: 11 franjas fortes, 25 coalescentes de franjas fortes, 30 franjas finas, 26 coalescentes de franjas finas e 14 detritos. A Figura 3 apresenta exemplos das cinco categorias e sua descrição.

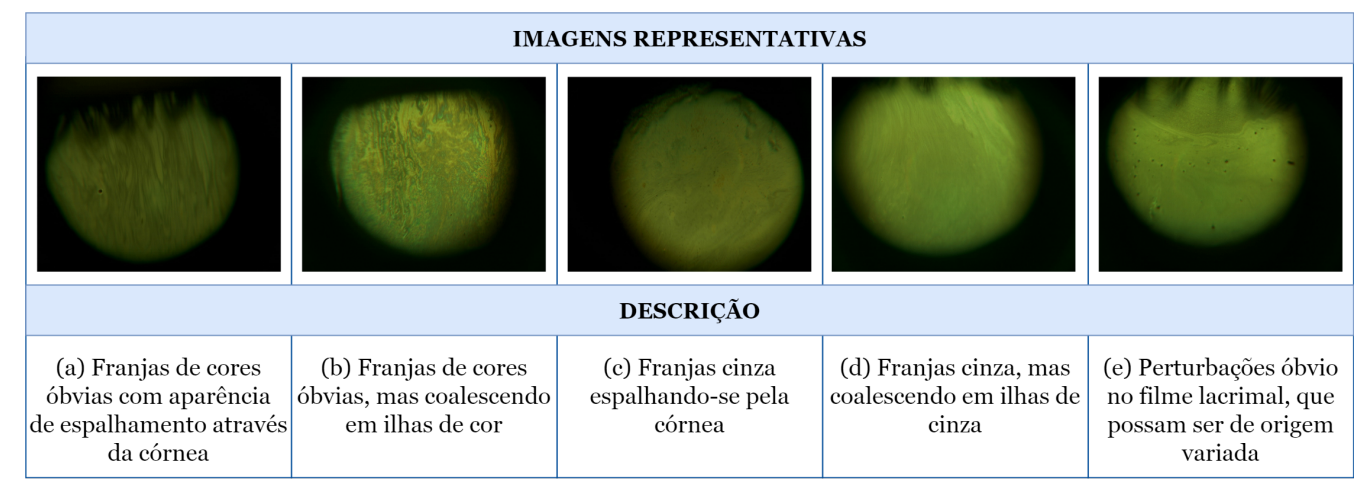

Figura 3. Categorias das imagens de interferometria: (a) Franjas fortes, (b) Coalescentes de franjas fortes, (c) Franjas finas, (d) Coalescentes de franjas finas (e) Detritos.

\subsection{Segmentação da ROI}

As imagens de entrada, conforme apresentado na Figura 4 (a), contêm informações irrelevantes presentes nas áreas externas. Assim, a parte central da área amarelada ou esverdeada, formada pela superfície anterior do filme lacrimal que cobre a córnea, demonstra ser a mais relevante da imagem. Portanto, realiza-se a extração da ROI seguindo a abordagem proposta em [Remeseiro et al. 2015].

A região relevante da imagem é caracterizada por tonalidades esverdeadas ou amareladas, assim apenas o canal G da imagem de entrada em RGB é considerado (Figura 4 
(b)). O fundo da imagem é determinado ao encontrar pixels cujo nível de cinza é inferior a um limite (Figura 4 (c)). Esse limite é calculado conforme a Equação 4:

$$
\text { limiar }=\text { media }-p \times \sigma
$$

onde media constitui o valor médio dos níveis de cinza da imagem, $\sigma$ é o desvio padrão e $p$ é um fator de peso empiricamente determinado $(p=0,1)$. Depois que a ROI preliminar é identificada, sua parte central deve ser localizada. Devido algumas imagens incluírem outras regiões irrelevantes, como cílios, o operador morfológico de erosão [Gonzalez and Woods 2008], usando uma elipse como elemento estruturante é aplicado (Figura 4 (d)). Em seguida, um retângulo dentro da região identificada acima é mapeado e reduzido até que nenhuma área do fundo permaneça (Figura 4 (e)) . Observa-se que o tamanho das imagens de entrada é 1280 x 1024 pixels, e o tamanho final da ROI é, em média, 547 x 578 pixels. A Figura 4 apresenta passo a passo a extração da ROI de uma imagem de entrada.

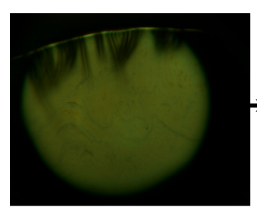

(a)

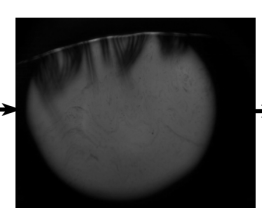

(b)

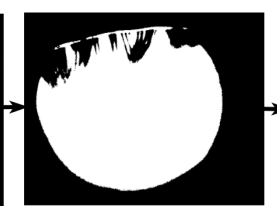

(c)

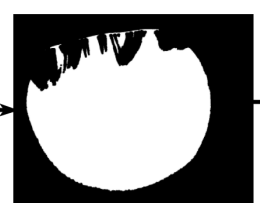

(d)

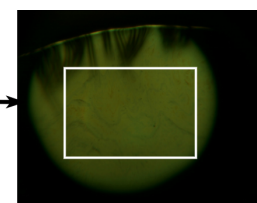

(e)

Figura 4. Etapas da segmentação da ROI.

\subsection{Extração de Características}

Essa etapa visa produzir medidas descritivas das imagens interferométricas, as quais formarão os vetores de características que serão usados na etapa de seleção e classificação. O procedimento de extração de características adotado neste trabalho é baseada na análise de textura.

$\mathrm{Na}$ descrição da textura foi utilizado a função K de Ripley, que é um método de análise de segunda ordem, aplicando nos espaços de cores baseado em cores oponentes, L*a*b*, HSV e RGB [Plataniotis and Venetsanopoulos 2013]. Para representação da imagem, foi utilizado o Local Binary Pattern (LBP) padrão [Ojala et al. 1996], com tamanho de janela 3x3, objetivando encontrar distribuição espacial de padrões locais da textura existente em análise. Esses procedimentos encontram-se detalhados nas próximas Seções.

\subsection{Estatística Espacial}

As abordagens em círculos e anéis (Seção 3) apresentadas sobre a função K de Ripley, são usadas nesse trabalho. Para ambas abordagens, os procedimentos adotados são semelhantes. Assim, para diferentes valores de raios $r$, a partir da escolha de um centro $i$, são analisados as ocorrências de LBPs de um mesmo valor $j$. Os padrões espaciais são examinados independentemente dos outros padrões e avaliados como a ocorrência ou não de um evento da distância $r$ definida.

Inicialmente, calcula-se o centro geométrico da ROI para cada imagem. Em seguida, é necessário calcular o maior raio possível baseado no centro determinado, ou seja, 
a distância do centro da ROI até o pixel mais distante pertencente à mesma. Foram utilizados 6 raios para a análise da variação de textura. Este parâmetro foi empiricamente testado, onde valores menores normalmente reduzem o desempenho e valores maiores não incluem características suficientes para serem analisadas.

Cada ROI é quantizada sucessivamente de 256 níveis, para 128, 64, 32, 16 e 8, correspondendo a 6 quantizações diferentes para cada um dos 6 raios de amostragem. $\mathrm{O}$ objetivo é encontrar características existentes em agrupamentos de um mesmo padrão, para representar a maior quantidade possível de informações sobre as imagens. Posteriormente, o LBP é aplicado sobre o resultado de cada quantização. Finalmente, cada raio de amostragem gera uma observação sobre cada um dos três canais de cada espaço de cores. Dessa forma, o modelo gera 3024 características $(6$ raios x $(256+128+64+32+16+$ 8)) para cada canal de cada espaço de cores.

\subsection{Seleção de Características}

A escolha do conjunto mínimo de características que tem o poder de discriminar as amostras é muito importante para simplificar o modelo e aumentar seu poder de generalização. Neste contexto, técnicas de reconhecimento de padrões são aplicadas para realizar seleção das características mais relevantes, a fim de aumentar a eficiência dos classificadores e reduzir custos computacionais.

Neste trabalho, as extrações de características realizadas pelas duas abordagens da função K de Ripley geram muitas variáveis. Cada abordagem adotada gera 3024 variáveis para cada canal de cada espaço de cores (3 x (3024)), totalizando 9072 variáveis. Portanto, essa etapa foi essencial para reduzir significativamente a grande quantidade de variáveis, e proporcionar um bom desempenho dos classificadores na etapa posterior.

Para efetuar este procedimento, foi utilizado o algoritmo Greedy Stepwise [Ambelu et al. 2010]. Esse algoritmo executa uma busca gulosa, no sentido forward ou backward, através do espaço de busca dos subconjuntos de atributos. A busca pode iniciar com nenhum/todos atributos ou de um ponto arbitrário no espaço. Encerra quando todas as inclusões/exclusões de atributos resultarem em uma diminuição na avaliação. Para tanto, este algoritmo encontra características que discriminam melhor as cinco classes do olho seco para a base de características usada. A aplicação do método gera um conjunto de variáveis para cada abordagem estudada. Esse conjunto contém menos redundâncias que poderiam prejudicar os classificadores durante as próximas etapas.

\subsection{Classificação}

Após a etapa de seleção de características, faz-se necessário o uso de técnicas de reconhecimento de padrões para classificar o conjunto das características selecionadas. Esse processo foi baseado na técnica de classificação supervisionada, que consiste em um processo prévio de treinamento de um classificador para o conhecimento dos padrões desejados.

As Redes Bayesianas são metodologias para a construção de sistemas que confiam no conhecimento probabilístico, e têm sido aplicadas em uma variedade de atividades no mundo. Portanto, os classificadores utilizados para classificar o conjunto de características selecionadas foram: Naive Bayes (NB), que é um classificador probabilístico que aplica o teorema de Bayes com pressupostos de independência, ou seja, suas características são assumidas como condicionalmente independentes, dada uma classe 
[Jensen 1996] e Bayes Net (BN), que é um modelo gráfico que representa um conjunto de variáveis e suas dependências condicionais [Nielsen and Jensen 2009]. Inicialmente, todos os valores das características foram normalizados para faixa de valores comuns entre 0 e 1, para melhorar o desempenho dos classificadores e padronizar a distribuição de valores das variáveis, as quais podem assumir diferentes domínios [Duda 1973].

As configurações para os dois classificadores foram as padrões fornecidas pelo WEKA [Hall et al. 2009]: a distribuição normal para os atributos numéricos e discretização supervisionada, para converter atributos numéricos para valores nominais, no NB; e o método K2 [Cooper and Herskovits 1992] foi o utilizado para pesquisar estruturas da rede, no $\mathrm{BN}$.

\subsection{Validação}

Depois da etapa de classificação, avalia-se o desempenho do método proposto. Assim, foram calculadas algumas estatísticas sobre os resultados dos testes.

O método proposto usa métricas comumente aplicadas em sistemas CADx e CAD para a análise de desempenho de sistemas baseados no processamento de imagens e reconhecimento de padrões, nomeadas: acurácia (Ac) [Duda 1973], desvio padrão (StdDev) em relação a acurácia e Kappa [Viera et al. 2005], aplicou-se também a curva $R e$ ceiver Operating Characteristic (ROC) [Fawcett 2006] como outra métrica de avaliação de desempenho das técnicas de detecção utilizadas. Devido desequilíbrio entre as classes, a medida F-Measure é usada, representando a média harmônica de precisão e recall [Fawcett 2006]. Finalmente, para o critério de validação, utilizou-se a validação cruzada $(k$-fold $=10)$ [Rodriguez et al. 2010], e após cinco classificações aleatórias foram calculados os valores médios para todas as métricas. Essa configuração de repetições objetivou mostrar que o método é robusto a diversas situações.

\section{Resultados e Discussões}

Nesta Seção, são apresentados os resultados obtidos pelo método proposto. Destaca-se que o propósito dos experimentos realizados é investigar a aplicabilidade do descritor de textura, para que possa contribuir com medidas discriminatórias dos padrões de interferência do filme lacrimal.

Para avaliar o desempenho do método, utilizou-se as métricas de validação (Seção 4.7). As 106 imagens de interferometria (Seção 4.1) foram, inicialmente, analisadas nos espaços de cores baseado em cores oponentes, L*a*b*, HSV e RGB. Posteriormente, extraíram-se 3024 características em cada um dos três canais em cada espaço de cores, usando a função K de Ripley e concatenando as variáveis, resultando em um total de 9072 variáveis por imagem.

Devido à grande quantidade de variáveis extraídas, o algoritmo de seleção Greedy Stepwise [Ambelu et al. 2010] foi utilizado para reduzir a dimensionalidade, e aumentar a eficiência dos classificadores. Esse algoritmo apresentou um alto poder discriminatório, resultando em boas variáveis nas duas abordagens exploradas pela função K de Ripley. As Tabelas 1 e 2, apresentam os resultados dos métodos utilizados. De cima para baixo, cada célula contém os resultados correspondentes respectivamente, aos classificadores (Clf) BN e NB na coluna Clf, em cada uma das métricas, dos espaços de cores. A última coluna 
mostra a quantidade de variáveis selecionadas (QVar) pelo algoritmo Greedy Stepwise para cada configuração analisada.

Tabela 1. Resultados da função K de Ripley usando abordagem em círculos.

\begin{tabular}{lccccccc}
\hline Espaços de Cores & Clf & Ac(\%) & StdDev(\%) & ROC & Kappa & F-Measure & QVar \\
\hline \multirow{2}{*}{ Cores Oponentes } & BN & $\mathbf{9 4 , 9 0}$ & $\mathbf{0 , 8 4}$ & $\mathbf{0 , 9 9}$ & $\mathbf{0 , 9 3}$ & $\mathbf{0 , 9 5}$ & \multirow{2}{*}{$\mathbf{1 1 6}$} \\
& NB & 94,15 & 0,78 & 0,99 & 0,92 & 0,94 & \\
L*a*b* & BN & 93,96 & 1,43 & 0,99 & 0,92 & 0,94 & 107 \\
\multirow{2}{*}{ HSV } & NB & 94,15 & 0,78 & 0,99 & 0,92 & 0,94 & \\
\multirow{2}{*}{ RGB } & BN & 92,64 & 2,25 & 0,99 & 0,90 & 0,92 & \multirow{2}{*}{87} \\
& NB & 92,83 & 1,43 & 0,99 & 0,90 & 0,92 & \\
& BN & 91,13 & 1,43 & 0,98 & 0,88 & 0,90 & \multirow{2}{*}{100} \\
\hline
\end{tabular}

A Tabela 1 apresenta a aplicação da abordagem em círculos. É possível verificar que o melhor resultado foi no espaço de cores baseado nas cores oponentes, utilizando o classificador BN após o processo de seleção de características pelo Greedy Stepwise, resultando em 116 variáveis selecionadas, do conjunto de 9072 geradas. O resultado apresenta 94,90\% de acurácia, 0,84\% de desvio padrão, 0,99 de ROC, 0,93 de Kappa e F-Measure de 0,95.

Tabela 2. Resultados da função K de Ripley usando abordagem em anéis.

\begin{tabular}{lccccccc}
\hline Espaços de Cores & Clf & Ac(\%) & StdDev(\%) & ROC & Kappa & F-Measure & QVar \\
\hline \multirow{2}{*}{ Cores Oponentes } & BN & 95,28 & 1,49 & 0,99 & 0,93 & 0,95 & \multirow{2}{*}{$\mathbf{1 3 3}$} \\
& NB & $\mathbf{9 5 , 6 6}$ & $\mathbf{1 , 8 3}$ & $\mathbf{0 , 9 9}$ & $\mathbf{0 , 9 3}$ & $\mathbf{0 , 9 5}$ & \\
L*a*b* & BN & 93,96 & 1,07 & 0,99 & 0,92 & 0,94 & \multirow{2}{*}{134} \\
& NB & 92,45 & 0,66 & 0,99 & 0,90 & 0,92 & \\
HSV & BN & 93,01 & 1,26 & 0,99 & 0,90 & 0,92 & \multirow{2}{*}{118} \\
RGB & NB & 91,69 & 1,81 & 0,99 & 0,89 & 0,91 & \\
& BN & 92,26 & 1,03 & 0,98 & 0,90 & 0,92 & \multirow{2}{*}{102} \\
\hline
\end{tabular}

O uso de anéis concêntricos elimina possíveis interferências de regiões centrais, fornecendo informações mais precisas a respeito das regiões periféricas da ROI. Assim, o melhor resultado obtido usando essa abordagem (Tabela 2) foi com as cores oponentes e o classificador NB, alcançando 95,66\% de acurácia, 1,83\% de desvio padrão, ROC de 0,99, Kappa 0,93 e F-Measure de 0,95, a partir de 133 variáveis selecionadas. Acredita-se que, a taxa de erro da acurácia de 4,34\% desse resultado ocorra devido à complexidade da base, que contém apenas 106 imagens em quantidade desproporcional entre as classes, e as suas texturas se comportarem de maneira semelhantes.

Analisando os resultados gerados pelos experimentos da função K de Ripley, observa-se que, a abordagem em anéis obteve o melhor resultado. Entretanto, as duas abordagens apresentam desempenho promissor e, em ambos, os resultados aplicando as cores oponentes superam os outros espaços de cores. Acredita-se que, as cores oponentes contêm informações mais relevantes que contribuem com o aumento dos resultados das métricas aplicadas. Além disso, concluiu-se que não existem diferenças muito significativas entre os dois classificadores, ambos apresentaram bons resultados. 


\subsection{Comparação com Trabalhos Relacionados}

Após a apresentação dos resultados, foi realizado uma análise comparativa dos resultados alcançados com os resultados dos trabalhos relacionados.

Na Tabela 3, é apresentado um resumo dos resultados encontrados nos trabalhos relacionados e em nosso método, utilizando a mesma base de imagens. Assim, pretendemos mostrar que nosso trabalho é promissor, sendo alcançados resultados superiores a 95\%, usando também análise de textura, mas aplicando a análise estatística com a função K de Ripley.

Tabela 3. Comparação com os trabalhos relacionados.

\begin{tabular}{llc}
\hline Trabalho & Técnicas & Ac(\%) \\
\hline [Villaverde et al. 2014] & $\begin{array}{l}\text { Estudo de diferentes métodos de combinação de } \\
\text { texturas e análise de cores }\end{array}$ & 91,51 \\
[Remeseiro et al. 2015] & $\begin{array}{l}\text { Análise de cores e texturas e o uso de métodos de } \\
\text { seleção de características }\end{array}$ & 93,40 \\
Método proposto & $\begin{array}{l}\text { Análise estatística baseado na função K de } \text { Ri- } \\
\text { pley }\end{array}$ & $\mathbf{9 5 , 6 6}$ \\
\hline
\end{tabular}

Analisando o melhor resultado alcançado com os apresentados na Tabela 3, podese notar que os resultados deste artigo são superiores. Portanto, os resultados apresentados mostram que as abordagens usadas na função K de Ripley produzem boas taxas de acerto, para classificação de imagens de interferometria do filme lacrimal. Acredita-se que, a combinação da abordagem em anéis com o espaço de cores baseado nas cores oponentes, identificam informações mais relevantes, discriminando melhor as categorias, resultando em boas taxas de acerto, e assim, comprovando a eficácia do método proposto para categorização da camada lipídica do filme lacrimal.

\section{Conclusão}

A diferenciação dos padrões de interferência da camada lipídica, em imagens de interferometria do filme lacrimal exclusivamente pela análise de textura, é uma tarefa difícil, principalmente pelo fato de ser muito comum as classes do olho seco possuírem características de textura semelhantes.

Este artigo apresentou um método para classificação das categorias da camada lipídica do filme lacrimal. Para alcançar esse objetivo, foram combinadas as abordagens estrutural (LBP) e estatística (K de Ripley) para análise da textura. No final, para categorizar a camada lipídica utilizaram-se os classificadores NB e BN.

Os resultados alcançados demonstraram desempenhos promissores na utilização de técnicas de análise de textura em imagens de interferometria, com base na função K de Ripley. Além disso, a representação da imagem por meio do LBP, foi um fator importante que contribuiu para a discriminação entre as categorias da camada lipídica do filme lacrimal. Embora a base de imagens utilizada seja complexa, mais testes com outras bases de imagens usando o interferômetro Doane (quando disponível), e usando Tearscope Plus, são necessários para melhorar o método, tornando-o mais robusto e genérico.

Como trabalhos futuros, pretende-se analisar a textura das imagens usando variações do LBP para geração dos padrões como, os algoritmos de LBP circular e de 
Completed Local Binary Pattern (CLBP), visto que, por serem circulares, estas abordagens analisam um número maior de vizinhos no cálculo do LBP. Além disso, avaliar outras técnicas de estatística espacial como, índice de Moran, de Getis e Coeficiente de Geary. Acredita-se que essas modificações podem proporcionar uma melhor discriminação das categorias do filme lacrimal.

\section{Agradecimentos}

Os autores agradecem a Coordenação de Aperfeiçoamento de Pessoal de Nível Superior (CAPES), o Conselho Nacional de Desenvolvimento Científico e Tecnológico (CNPq), e a Fundação de Amparo à Pesquisa e ao Desenvolvimento Científico e Tecnológico do Maranhão (FAPEMA) pela ajuda financeira.

\section{Referências}

Ambelu, A., Lock, K., and Goethals, P. (2010). Comparison of modelling techniques to predict macroinvertebrate community composition in rivers of ethiopia. Ecological Informatics, 5(2):147-152.

Çesmeli, E. and Wang, D. (2001). Texture segmentation using gaussian-markov random fields and neural oscillator networks. IEEE Transactions on neural networks, 12(2):394-404.

Cooper, G. F. and Herskovits, E. (1992). A bayesian method for the induction of probabilistic networks from data. Machine learning, 9(4):309-347.

Dash, M. and Liu, H. (2003). Consistency-based search in feature selection. Artificial intelligence, 151(1-2):155-176.

Dean, J. (2014). Big data, data mining, and machine learning: value creation for business leaders and practitioners. John Wiley \& Sons.

Doane, M. G. (1989). An instrument for in vivo tear film interferometry. Optometry and Vision Science, 66(6):383-388.

Doi, K. (2007). Computer-aided diagnosis in medical imaging: historical review, current status and future potential. Computerized medical imaging and graphics, 31(4-5):198211.

Duda, R. (1973). Pattern classification and scene analysis. New-York, London, Sydny, Tronto A Wiley-Interscience Publication.

Fawcett, T. (2006). An introduction to roc analysis. Pattern recognition letters, 27(8):861-874.

Gonzalez, R. and Woods, R. (2008). Digital image processing. Pearson, Prentice Hall.

Hall, M., Frank, E., Holmes, G., Pfahringer, B., Reutemann, P., and Witten, I. H. (2009). The weka data mining software: an update. ACM SIGKDD explorations newsletter, 11(1):10-18.

Hall, M. A. (1999). Correlation-based feature selection for machine learning. University of Waikato Hamilton.

Jensen, F. V. (1996). An introduction to Bayesian networks, volume 210. UCL press London. 
Lancaster, J. and J Downes, B. (2004). Spatial point pattern analysis of available and exploited resources. Ecography, 27(1):94-102.

Martins, L. O. (2007). Detecção de massas em imagens mamográficas através do algoritmo growing neural gas e da função k de ripley. Master's thesis, Universidade Federal do Maranhão.

Nielsen, T. D. and Jensen, F. V. (2009). Bayesian networks and decision graphs. Springer Science \& Business Media.

Ojala, T., Pietikäinen, M., and Harwood, D. (1996). A comparative study of texture measures with classification based on featured distributions. Pattern recognition, 29(1):5159.

Plataniotis, K. N. and Venetsanopoulos, A. N. (2013). Color image processing and applications. Springer Science \& Business Media.

Remeseiro, B., Bolon-Canedo, V., Peteiro-Barral, D., Alonso-Betanzos, A., GuijarroBerdinas, B., Mosquera, A., Penedo, M. G., and Sánchez-Marono, N. (2014). A methodology for improving tear film lipid layer classification. IEEE journal of biomedical and health informatics, 18(4):1485-1493.

Remeseiro, B., Oliver, K. M., Tomlinson, A., Martin, E., Barreira, N., and Mosquera, A. (2015). Automatic grading system for human tear films. Pattern Analysis and Applications, 18(3):677-694.

Ripley, B. D. (1977). Modelling spatial patterns. Journal of the Royal Statistical Society. Series B (Methodological), pages 172-212.

Rodriguez, J. D., Perez, A., and Lozano, J. A. (2010). Sensitivity analysis of k-fold cross validation in prediction error estimation. IEEE transactions on pattern analysis and machine intelligence, 32(3):569-575.

Rolando, M. and Zierhut, M. (2001). The ocular surface and tear film and their dysfunction in dry eye disease. Survey of ophthalmology, 45:S203-S210.

Valim, V., Trevisani, V. F. M., de Sousa, J. M., Vilela, V. S., and Belfort, R. (2015). Current approach to dry eye disease. Clinical reviews in allergy \& immunology, 49(3):288297.

Vapnik, V. N. (1999). An overview of statistical learning theory. IEEE transactions on neural networks, 10(5):988-999.

Viera, A. J., Garrett, J. M., et al. (2005). Understanding interobserver agreement: the kappa statistic. Fam Med, 37(5):360-363.

Villaverde, D. G., Remeseiro, B., Barreira, N., Penedo, M. G., and González, A. M. (2014). Feature selection applied to human tear film classification. In ICAART (1), pages $395-402$.

VOPTICAL_GCU, V. (2017). Optical dataset acquired and annotated by optometrists from the department of life sciences, glasgow caledonian university (uk), 2013. http://www.varpa.org/voptical_gcu.html.

Zhao, Z. and Liu, H. (2007). Searching for interacting features. In IJCAI, volume 7, pages 1156-1161. 